\title{
optique adaptative extrême pour la détection de planètes extrasolaires
}

\author{
Thierry FUSCO',2, Jean-François SAUVAGE ${ }^{1,2,3}$, Cyril PETIT' ${ }^{1}$, Kjetil DOHLEN² ${ }^{2}$ Anne COSTILLE², David MOUILLET ${ }^{3}$ ef J.-L. BEUZIT ${ }^{3}$ \\ 'ONERA the French Aerospace Lab. \\ ${ }^{2}$ Aix Marseille Université, CNRS, LAM \\ ${ }^{3}$ Univ. Grenoble Alpes, CNRS, IPAG \\ thierry.fusco@onera.fr
}

SPHERE est le tout nouveau « chasseur de planètes » de l'Observatoire Européen Austral. Installé en mai 2014 sur l'un des quatre télescopes géants du Very Large Telescope sur le Cerro Paranal (Chili), il atteint, grâce à son système d'optique adaptative extrême $S A X O$, des résolutions angulaires sans précédent que ce soit au sol ou dans l'espace.

'instrument SPHERE (spectro-polarime-

L trichigh-contrastexoplanet research) [1] a pour but la détection et la caractérisation d'objets faiblement lumineux, comme des planètes géantes gazeuses, à proximité d'étoiles brillantes. Cet exploit, qui est équivalent à détecter, depuis Paris, la lumière d'une bougie située à $50 \mathrm{~cm}$ d'un phare dans la rade de Marseille, nécessite I'utilisation conjointe de technologies optiques de pointe, toutes à la limite de l'état de l'art international. En particulier, au cœur de l'instrument, bat plus de 1200 fois par seconde un système d'optique adaptative appelé SAXO (sphere adaptive-optics for exoplanet observation) [2, 3]. Son rôle est de corriger tous les défauts de phase (et donc toutes les distorsions de l'image) introduits par la turbulence atmosphérique mais aussi par le télescope et l'instrument lui-même. SAXO fournit alors aux instruments scientifiques un front d'onde quasi parfait et permet donc de former des images limitées par la diffraction du télescope aux longueurs d'onde infrarouges et visibles, une performance sans précédent sur un télescope de ce diamètre.

L'instrument SPHERE a été conçu et réalisé par un consortium européen piloté par I'Institut de Planétologie et Astrophysique de Grenoble et comprenant I'ONERA, le Laboratoire d'Astrophysique de Marseille, le Laboratoire d'Études Spatiales et

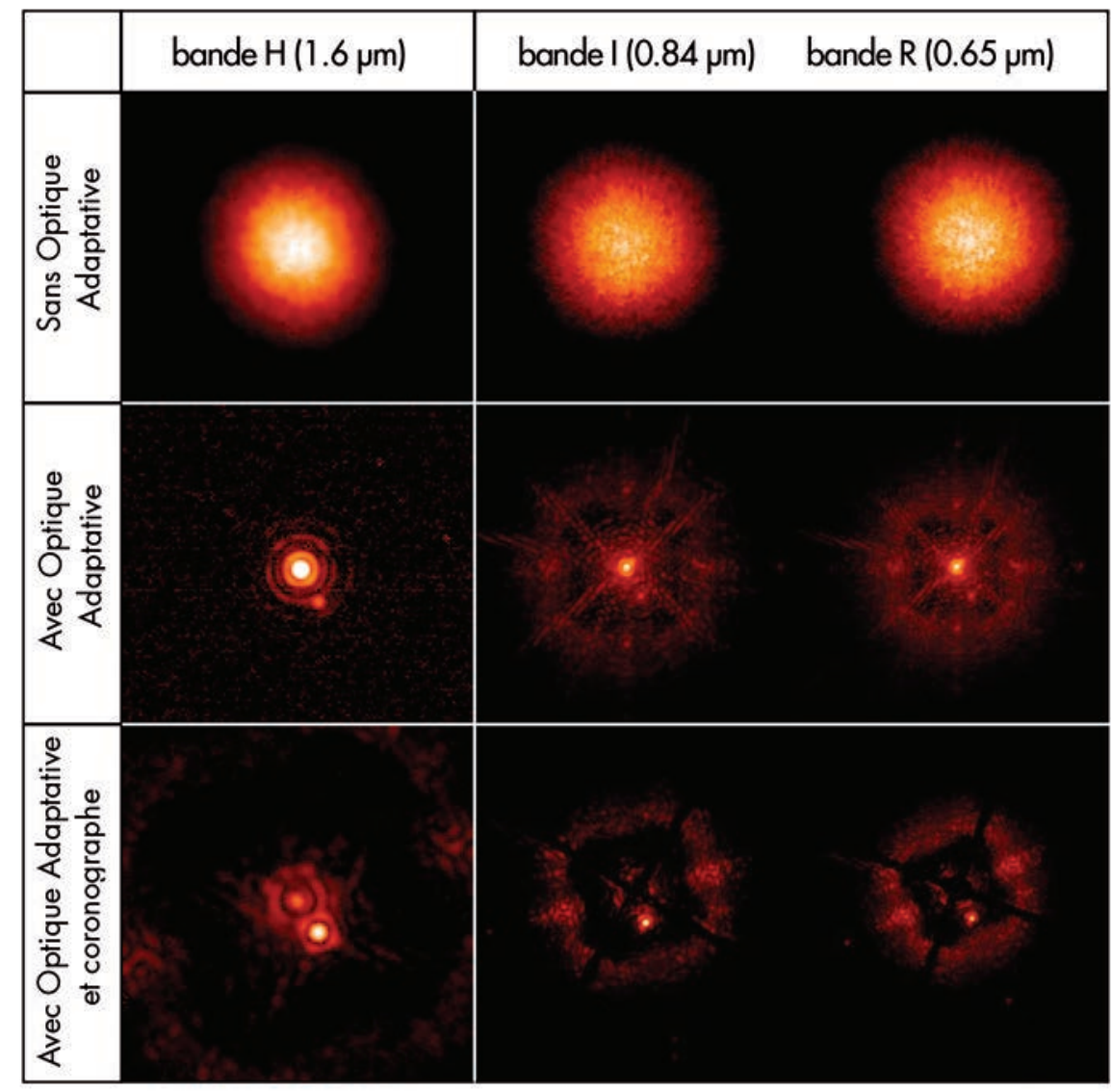

Figure 1. Illustration des différents étages de corrections de SPHERE. De haut en bas : images non corrigées, corrigées par $\mathrm{OA}$ et images derrière le coronographe. De gauche à droite : trois bandes spectrales $\mathrm{H}$, I et R. Le rapport de Strehl en $\mathrm{H}$ est supérieur à $90 \%$ et la limite de diffraction est atteinte en I et $\mathrm{R}$ (soit moins de 20 millisecondes d'arc dans le dernier cas). L'OA permet de séparer l'étoile de son compagnon et la coronographie permet d'éliminer la majeure partie de la contribution de l'étoile pour gagner en contraste sur le compagnon. 
d'Instrumentation en Astrophysique, le laboratoire Lagrange ainsi que des instituts allemands, italiens, suisses et néerlandais, en collaboration avec l'ESO.

En mai 2014, SHERE (figure 2) a été installé sur le $3^{e}$ télescope de 8 mètres (Melipal) du Very Large Telescope de l'Observatoire Européen Austral, au Chili. Après plus de 3 ans de tests exhaustifs (performances ultimes, robustesse, opérabilité) réalisés en Europe puis 4 mois de remise en route au Chili, l'instrument est maintenant ouvert à la communauté astronomique depuis début 2015.

\section{SPHERE}

Afin de répondre aux attentes toujours plus exigeantes des astronomes, l'instrument SPHERE combine de nombreux systèmes optiques complexes et des processus de traitement d'images optimisés afin d'isoler les quelques photons provenant de l'objet d'intérêt parmi les dizaines de millions provenant de son étoile hôte. D'abord une optique adaptative (OA, voir Photoniques $n^{\circ} 58$, mars-avril 2012) de pointe met en forme le faisceau en le corrigeant de toutes ses imperfections (erreurs de pointage, vibrations, aberrations instrumentales quasi-statiques et turbulence atmosphérique) avec une précision nanométrique.
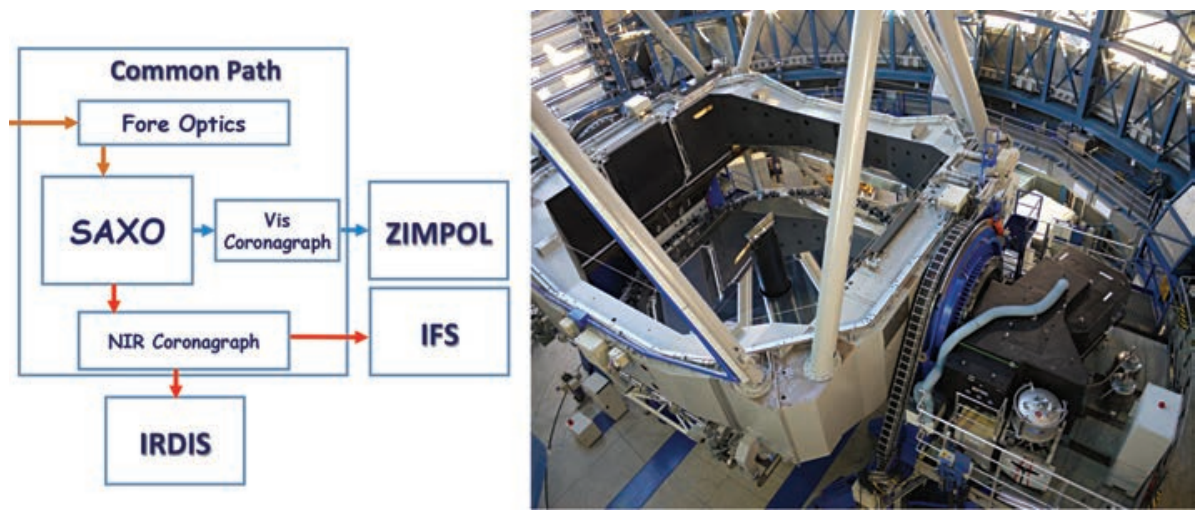

Figure 2. À gauche : concept global de SPHERE indiquant les 4 sous-systèmes et les principales fonctionnalités de l'instrument. À droite : SPHERE au foyer Nasmyth du télescope Melipal : $6 \times 4 \times 2,5 \mathrm{~m}^{3}$, 8 tonnes, le tout reposant sur 3 piliers anti-vibrations. Un monstre à la finesse d'image et à la stabilité optique inégalées.

Un coronographe est ensuite utilisé pour éliminer les photons provenant de l'étoile, soit en les bloquant physiquement à l'aide d'une pastille opaque, soit en créant des interférences destructives au niveau du plan focal de l'instrument.

En aval de ce premier étage d'optimisation du faisceau optique, trois détecteurs vont analyser la lumière résiduelle non bloquée par les coronographes afin d'en extraire une information pertinente sur l'environnement de l'étoile observée (voir figure 1).

- IRDIS [4], une caméra d'imagerie sensible dans l'infrarouge (entre 0,95 et 2,3 microns) permettant d'imager simultanément la même scène à deux longueurs d'onde proches pour bénéficier d'une potentielle information spectrale dans l'objet observé. IRDIS a, en plus de ses capacités d'imagerie, un mode spectrographe à fente et un mode polarimétrique.

- IFS [5], un spectrographe imageur lui aussi sensible dans le proche infrarouge (entre 0,95 et 1,7 micron) avec une résolution spectrale de 40, qui va permettre une analyse fine des objets et l'utilisation de techniques de traitement de données avancées pour gagner significativement en performances et potentiellement détecter des objets/planètes plusieurs millions de fois moins lumineux que leur étoile hôte. On notera que 80 \% des observations seront effectuées avec IRDIS et IFS fonctionnant de concert dans

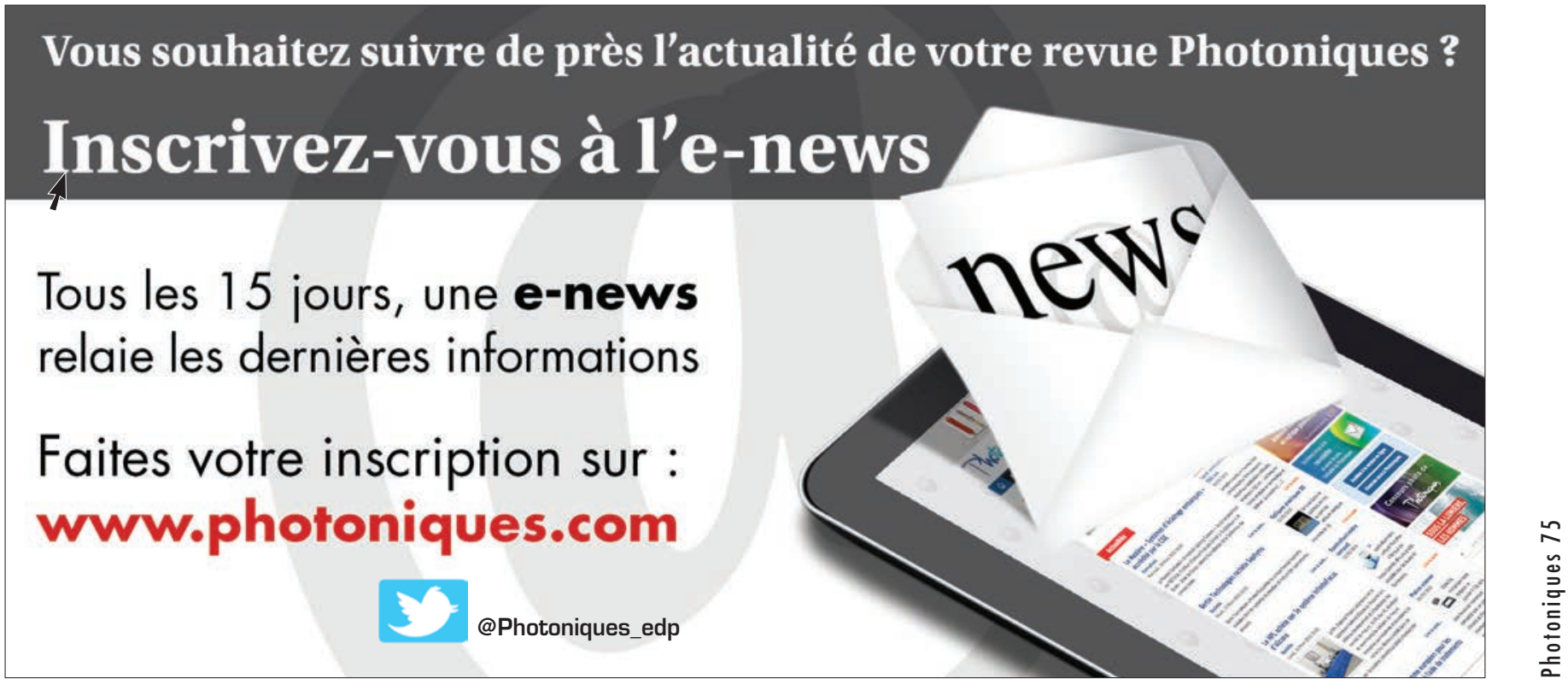


deux sous bandes distinctes de l'infrarouge proche.

- ZIMPOL [6], une caméra visible (entre 0,5 et 0,9 microns) qui permet d'acquérir simultanément (et très rapidement) des images soit à deux longueurs d'onde différentes soit dans des états de polarisation différents. Là encore, l'idée est d'utiliser des possibles fluctuations de la polarisation de la lumière reçue pour distinguer entre les photons résiduels provenant de l'étoile, non arrêtés par le coronographe à cause des imperfections du système, qui ne sont pas polarisés et ceux réfléchis par l'objet d'intérêt qui, eux, peuvent l'être.

Le concept de cet instrument aux multiples caractéristiques et fonctionnalités (qui en fait l'un des instruments les plus complexes jamais installé sur le VLT) est schématisé à la figure 2 avec une photo de l'instrument installé au foyer Nasmyth de Melipal, le $3^{\mathrm{e}}$ télescope de 8 mètres du VLT et avec une illustration des différentes étapes de mise en forme et traitement du faisceau optique : correction par OA, coronographie et détection par un instrument scientifique.

\section{SAXO}

SAXO (sphere adaptive-optics for exoplanet observation) est le cœur de SPHERE, un cœur qui bat 1200 fois par seconde pour mesurer et corriger tous les défauts du front d'onde provenant de l'objet d'intérêt afin d'assurer les meilleures qualités d'image et résolutions angulaire pour un télescope monolithique qu'il soit situé au sol ou dans l'espace.

Cette prouesse technologique repose sur la combinaison de concepts et de composants parmi les plus avancés au monde. SAXO utilise un miroir de pointage rapide et de grande dimension $(50 \mathrm{~mm}$ à $800 \mathrm{~Hz}$ ) fourni par l'observatoire de Paris, un miroir déformable (DM) de 1377 actionneurs fourni par la société CILAS et un miroir actif torique, dédié à la compensation des aberrations propres, lentement variables du DM, fourni par le Laboratoire d'Astrophysique de Marseille [7].

L'analyse de front d'onde [8] se base sur un analyseur de type Shack-Hartmann (SH) utilisant une caméra EMCCD (electron-multiplying (CD) de $240 \times 240$ pixels avec une fréquence trame de $1200 \mathrm{~Hz}$ et un bruit de lecture équivalent (après amplification du signal) inférieur à 0,1 électron. Cette technologie a depuis été améliorée et distribuée par une PME Marseillaise (First Light Imaging). Un filtre spatial ajustable en amont du SH permet de s'affranchir du phénomène de repliement. Les performances pointues du détecteur de SPHERE alliées aux diverses optimisations de concepts et de traitement de données du SH permettent à SAXO d'atteindre des magnitudes limites allant bien au-delà des spécifications originales et donc d'ouvrir la voie à l'observation de nombreux objets qui avaient été estimés inaccessibles lors de la définition de l'instrument (figure 3).

L'analyseur de front d'onde et les optiques déformables sont reliés par un calculateur temps réel [9] développé par l'ESO, baptisé SPARTA. Il contrôle l'ensemble des optiques déformables de SAXO avec un temps de latence inférieur à 80 microsecondes. II identifie et s'adapte automatiquement aux caractéristiques principales des défauts à corriger. Un filtrage de Kalman permet de compenser jusqu'à 12 pics de vibrations qui pourraient apparaître aléatoirement entre 10 et $300 \mathrm{~Hz}$. Cette dernière fonctionnalité assure d'atteindre un bouger résiduel de l'image inférieur à 2 millisecondes d'arc (soit moins d'un vingtième de la limite de diffraction en bande $\mathrm{H}$ ) et permet aux différents coronographes de fonctionner avec leurs performances ultimes.
En plus des composants " classiques » d'un système d'OA, SAXO se distingue par la présence de deux boucles de contrôle auxiliaires qui assurent la stabilité globale de l'ensemble de l'instrument:

- un centrage ultra précis (inférieur à 0,5 milliseconde d'arc, soit un centième de la diffraction en $\mathrm{H}$ ) fonctionnant à $1 \mathrm{~Hz}$ obtenu grâce à un senseur de tip-tilt infrarouge spécifique situé au plus près des coronographes et un miroir de tilt différentiel [10];

- un centrage fin de la pupille [11] (avec une précision de 2 pour mille) toutes les 10 secondes pour compenser les dérives du télescope lui-même.

Toutes ces fonctionnalités rassemblées et optimisées en temps réel durant l'observation permettent d'obtenir une correction quasi-parfaite du front d'onde avec des performances sans précédent sur un télescope de 8 mètres : plus de $90 \%$ de rapport de Strehl en bande $\mathrm{H}$ et des images limitées par la diffraction aux longueurs d'onde du visible (I et R) permettant à SPHERE d'atteindre ses objectifs en termes de contraste et de limite de détectivité. De plus, SAXO a été dimensionné et réalisé pour être aussi automatique que possible, c'est-à-dire qu'il doit s'optimiser en utilisant ses données internes sans intervention extérieure. Dans le même esprit, les processus d'étalonnage et de vérification des performances sont complètement automatiques. Ainsi SAXO, et plus généralement SPHERE, peut être opéré par un non-spécialiste sans aucune perte en performance.

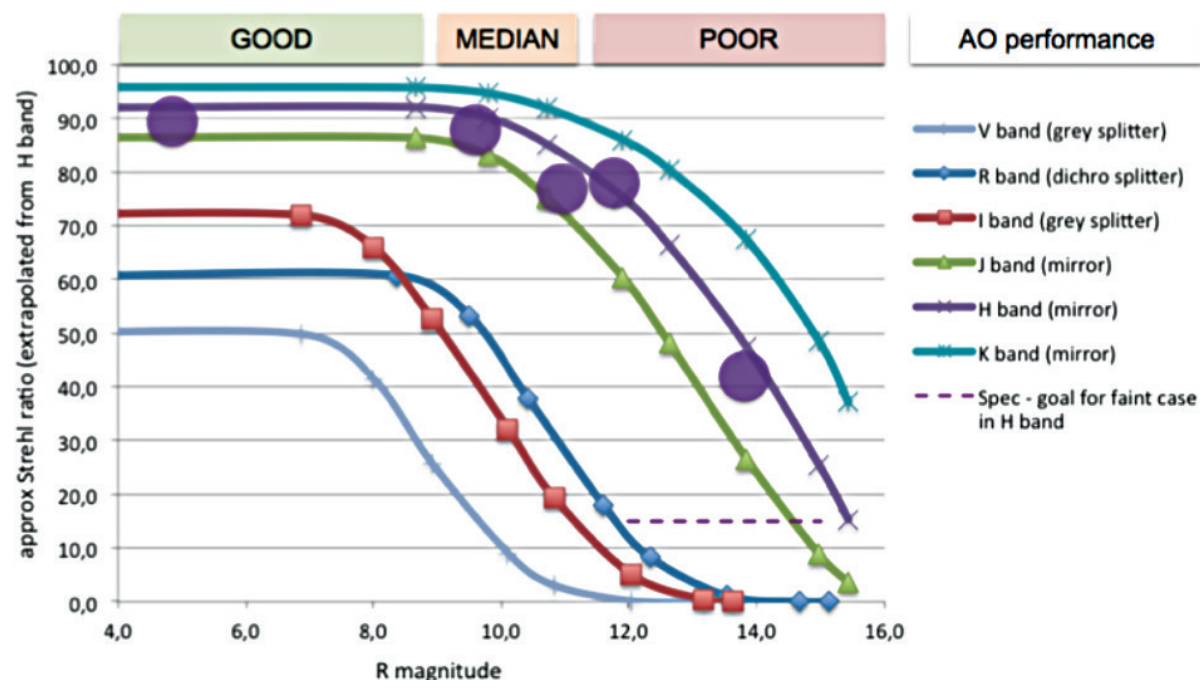

Figure 3. Performances de SAXO mesurées au laboratoire (courbes) et sur le ciel (points). 


\section{Du VLT à l'E-ELT}

Dans les prochaines années SPHERE va, sans nul doute, apporter des percées significatives sur la compréhension de la formation et l'évolution des systèmes planétaires, mais il peut et doit aussi être vu comme un précurseur pour la future instrumentation du télescope européen géant de 39 mètres (voir Photoniques $\mathrm{n}^{\circ} 48$, septembre-octobre 2010) qui va être construit sur le Cerro Armazones (Chili), à une vingtaine de kilomètres du VLT. L'expérience acquise sur SPHERE (tant sur le dimensionnement que sur son opération) sera la base sur laquelle les futurs instruments seront bâtis. Des instruments toujours plus puissants et performants pour atteindre, à l'horizon 2030, l'objectif ultime : enregistrer et analyser la lumière provenant de planètes rocheuses situées dans une zone propice à l'émergence de la vie.

\section{Pour en savoir plus}

[1] J-L. Beuzit, D. Mouillet, C. Moutou, K. Dohlen, P. Puget, T. Fusco, A. Boccaletti, "A planet finder instrument for the VLT", in Proceedings of IAU Colloquium 200, Direct Imaging of Exoplanets: Science \& Techniques (Cambridge University Press, 2005), pp. 317-323

[2] T. Fusco et al., "High-order adaptive optics requirements for direct detection of extrasolar planets: Application to the SPHERE instrument", Optics Express 14, 7515-7534 (2006)

[3] T. Fusco et al., "Final performance and lesson-learned of SAXO, the VLT-SPHERE extreme AO: from early design to on-sky results", Proc. SPIE 9148, Adaptive Optics Systems IV, 91481U (7 August 2014)

[4] K. Dohlen et al., "The infra-red dual imaging and spectrograph for SPHERE: design and performance", 7014, jul 2008, SPIE

[5] R. Claudi et al., "The SPHERE IFS at work", Proc. SPIE 9147, Ground-based and Airborne Instrumentation for Astronomy V, 91471L (8 July 2014)

[6] R. Roelfsema et al., "The ZIMPOL high contrast imaging polarimeter for SPHERE: system test results", Proc. SPIE 9147, Ground-based and Airborne Instrumentation for Astronomy V, $91473 \mathrm{~W}$ (8 July 2014)

[7] Hugot et al., "Active optics methods for exoplanets direct imaging", A\&A 538, A139 (2012)

[8] J.-F. Sauvage et al., "Wave-front sensor strategies for SPHERE: first on-sky results and future improvements", Proc. SPIE 9148, Adaptive Optics Systems IV, 914847 (14 November 2014)

[9] C. Petit et al., "SPHERE eXtreme AO control scheme: final performance assessment and on sky validation of the first auto-tuned LQG based operational system", Proc. SPIE 9148, Adaptive Optics Systems IV, 914800 (7 August 2014)

[10] P. Baudoz et al., "The differential tip-tilt sensor of SPHERE", Proc. SPIE 7735, Ground-based and Airborne Instrumentation for Astronomy III, 77355B (21 July 2010)

[1 1 ] G. Montagnier, T. Fusco, J.-L. Beuzit, D. Mouillet, J. Charton, L. Jocou, Pupil stabilization for SPHERE's extreme AO and high performance coronagraph system, Opt. Express 15, 15293-15307 (2007)

\section{Découvrez l'Annuaire des formations en optique photonique}

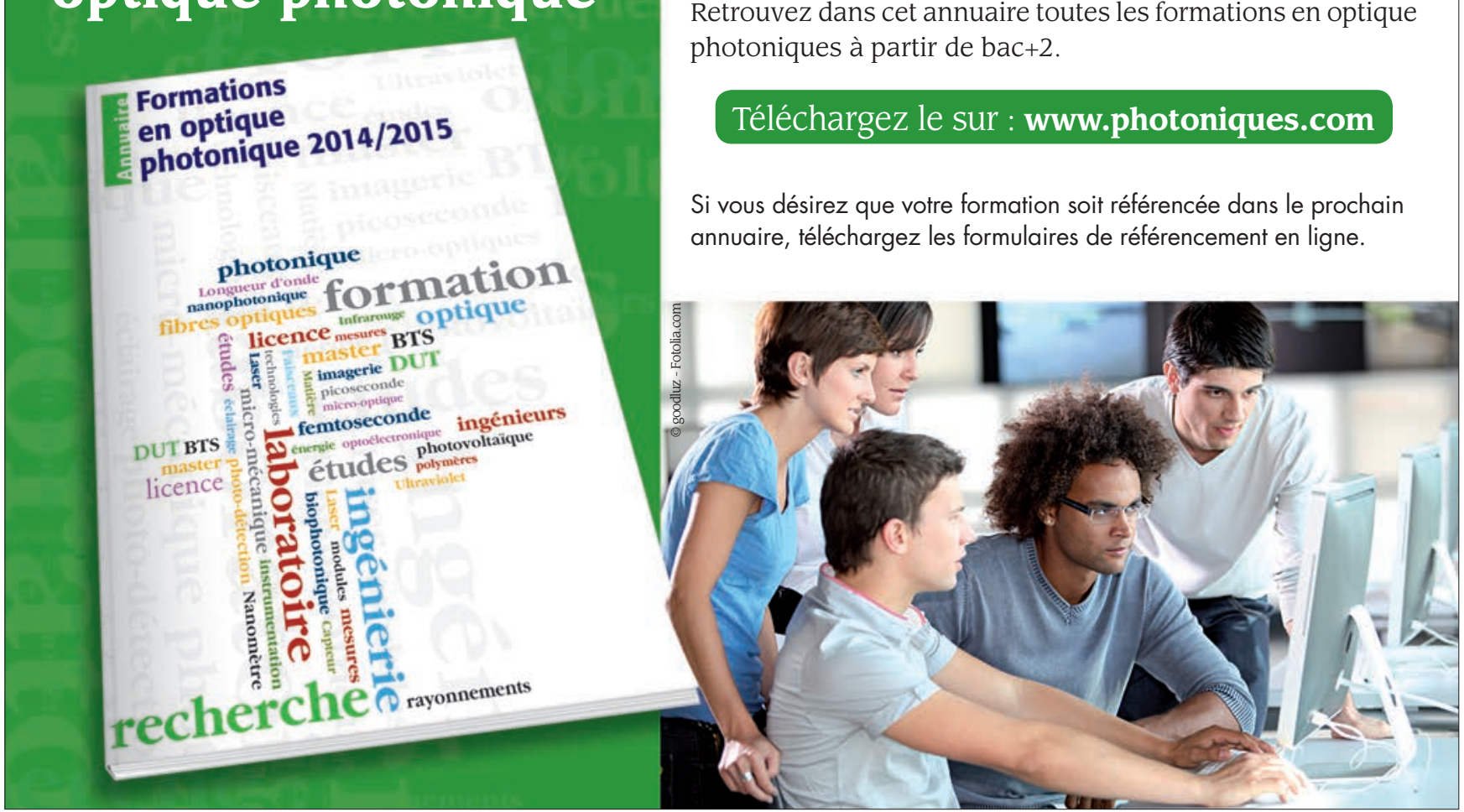

L'annuaire des formations en optique photonique est destiné aux responsables RH des entreprises et aux futurs étudiants.

Retrouvez dans cet annuaire toutes les formations en optique a partir de bac+2

\section{Téléchargez le sur : www.photoniques.com}

